\title{
Non-Invasive Evaluation of Bladder Outlet Obstruction in Men Suspected of Benign Prostatic Hyperplasia: Usefulness of the D Index
}

\author{
Françoise A. Valentinia ${ }^{a}$ b Pierre P. Nelson ${ }^{a} \quad$ Philippe E. Zimmern ${ }^{c}$ \\ ${ }^{a}$ ER6-Université Pierre et marie Curie; ${ }^{b}$ Physical Medicine and Rehabilitation, Hôpital Rothschild, Paris, France; \\ 'Department of Urology, UT Southwestern Medical Center, Dallas, Tex., USA
}

\section{Key Words}

Bladder outlet obstruction - Benign prostatic hyperplasia • Voiding dysfunction index • Free uroflow •

Non invasive testing

\begin{abstract}
Objective: To compare a new index of voiding dysfunction (D) based purely on free uroflow vs. Abrams-Griffiths (A-G) number obtained from intubated flow, for classification of bladder outlet obstruction in men. Patients and Methods: Urodynamic tracings of 60 non-neurological patients ( 30 before transurethral resection of the prostate and of 30 men suspected of benign prostatic hyperplasia included in a medical therapy trial) were retrospectively analyzed. The Valentini-Besson-Nelson model was used to evaluate the value of the $D$ index. A-G was obtained from intubated flows. Obstruction was defined as $\mathrm{D}>32.5 \mathrm{~cm} \mathrm{H}_{2} \mathrm{O}$ (translation of A-G criterion). Results: D showed $82.05 \%$ sensitivity with $66.66 \%$ specificity; the positive predictive value was $82.05 \%$ and the negative predictive value $66.66 \%$ for the whole population, 83.3 vs. $80.9 \%$ sensitivity with 58.3 vs. $77.7 \%$ specificity for pre- transurethral resection of the prostate vs. medical therapy group. Conclusion: $\mathrm{D}$ index which can be obtained from a free uroflow appears as a valuable alternative to invasive urodynamic investigations when the diagnosis of bladder outlet obstruction needs to be more solidly established before a treatment decision or in men suspected of benign prostatic hyperplasia who elect for watchful waiting.
\end{abstract}

Copyright $\odot 2012$ S. Karger AG, Basel

\section{KARGER}

Fax +4161306 1234

E-Mail karger@karger.ch

www.karger.com
(C) 2012 S. Karger AG, Basel

1015-9770/12/0063-0124\$26.00/0

Accessible online at:

www.karger.com/cur

\section{Introduction}

The "gold standard" for evaluation of bladder outlet obstruction (BOO) in adult males is the use of pressureflow (PF) studies [1]. From PFs, the best methods for the diagnosis of BOO are the "provisional ICS nomogram" which implies the location of a critical point of coordinates maximum flow rate Qmax and detrusor pressure at Qmax $\left(\mathrm{p}_{\text {det.Qmax }}\right)$ and the Abrams-Griffiths (A-G) number [1] derived from the coordinates of that critical point. The main concern is that the urodynamic study necessary to extract Qmax and $\mathrm{P}_{\text {detQmax }}$ is invasive, and implies additional time and costs. In current practice, a free uroflow (FF) is frequently used as a screening test despite its lack of objective evaluation of the lower urinary tract dysfunction. FF is a very simple test, easy to perform and cheap. Thus, a first question was: can we obtain information on $\mathrm{BOO}$ and on the risk of acute urinary retention from the only FF? Using a mathematical model of micturition (the Valentini-Besson-Nelson (VBN) model) a new index of voiding dysfunction (D) based purely on FF has been developed to assist in the office management of patients with benign prostatic hyperplasia (BPH) [2]. A second question was: can we propose a simple device allowing an accurate screening of BOO from a FF recording? In a previous study [3] it has been shown that $\mathrm{D}$ values from FF or intubated PF were similar preoperatively but markedly reduced after transurethral resection of the prostate (TURP). This more stable index compared to Qmax may offer an alternative approach to gauge the voiding per-

Françoise A. Valentin

Physical Medicine and Rehabilitation

Hôpital Rothschild

5 , rue Santerre

FR-75012 Paris (France)

E-Mail francoise.valentini@rth.aphp.fr; favalentini@gmail.com 
formance of a symptomatic patient with benign prostatic hyperplasia and possible BOO. Thus a simple device associates a flow meter and a "click and read" version of the $\mathrm{VBN}^{\circledR}$ software.

To further our previous results, we studied the specificity and sensibility of the D index when compared to the provisional International Continence Society method (based on A-G number) for classification of BOO in a short series of men scheduled for either medical or surgical treatments.

\section{Patients and Methods}

\section{VBN Model}

The VBN micturition model [4] is a quantitative description of the phenomena governing micturition; these phenomena are contractility, elasticity and visco-elasticity of the bladder [5], elasticity of the urethra [6], compression of the urethra by the sphincter, hydrodynamics of a turbulent incompressible fluid [7] and nervous control of both detrusor and sphincter [8]. Each phenomenon considered separately is accurately described and can be easily studied. However, when combined as during voiding, they constitute such an intricate set that to precisely analyze micturition, elaborate software is needed. The $\mathrm{VBN}^{\circledR}$ software allows to deduct the effects (changes observed in the uroflow curve) from the causes (changes in the voiding parameters) and vice versa. As explained in a prior publication [4], the VBN calculations need only a set of simple entries including gender, initial bladder volume, and catheter diameter for PF.

Two VBN parameters, $k$ and pucp, describe the obstructive status of a BOO patient. $k$ is the "detrusor force parameter" which is equal to 1 for a normal detrusor, $>1$ in the initial phase of obstruction (significant hypertrophy with maintained contractility) and $<1$ in the decompensation phase. pucp is the "prostatic urethral counter pressure" which models the compressive effect of the enlarged prostate on the urethra and has a unit in $\mathrm{cm}_{2} \mathrm{O}$. These 2 mechanical parameters remain constant during the same session or between sessions PF the BPE condition remains fairly stable. pucp is strongly correlated [9] with the Abrams-Griffiths [1] and the obstruction coefficient [10] numbers; $k$ is strongly correlated [9] with the Watt-Factor [11] and a modified projected isometric pressure (m-PIP) [9].

\section{The D Index}

A couple of VBN parameters (pucp, $k$ ) characterize the obstructive status of a patient. The values of these 2 parameters cannot be evaluated from only a FF because during a FF the detrusor pressure is not recorded. However it has been demonstrated that one given FF is associated with a limited set of detrusor pressure curves (fig. 1a), implying a limited set of couples (pucp, $k$ ). Furthermore, all these couples (pucp, $k$ ) are not scattered in the plane (pucp, $k$ ) but located on only one curve (fig. 1b). Each curve is referenced by a critical point $\mathrm{D}$ which is chosen as the value of pucp for $k=1$ (normal detrusor) (fig.1b). Thus D can be deduced from any FF or PF (as opposed to Qmax). It characterizes the obstructive status of the patient. In summary, D is a measured

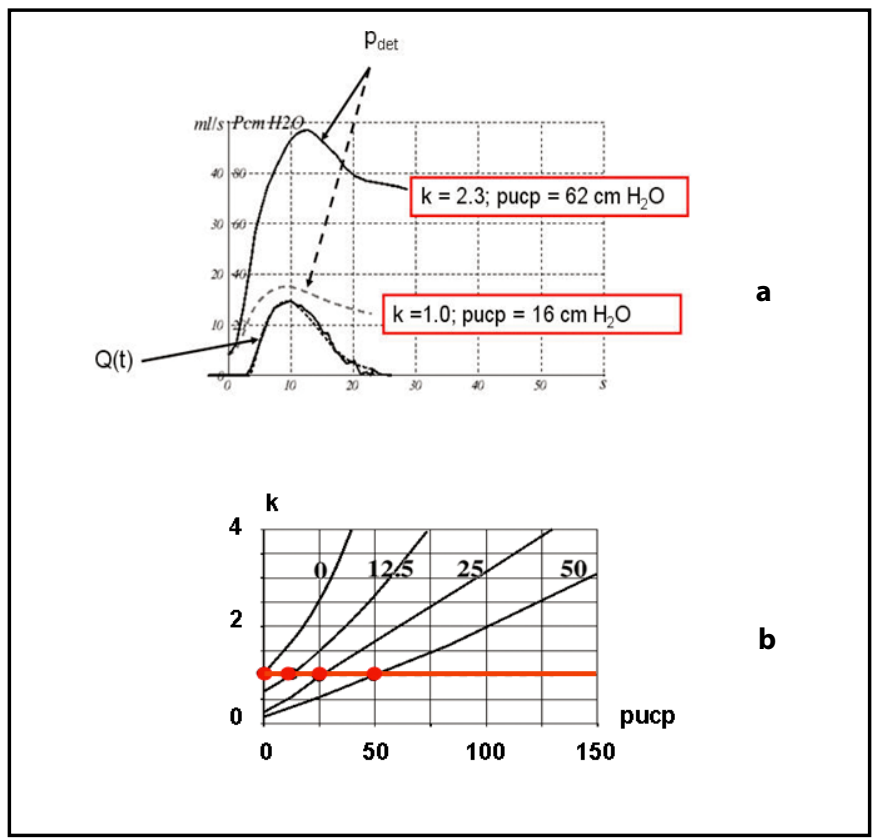

Fig. 1. a Modelled analysis of a free uroflow $Q(t)$ of a BPE patient. Recorded curve: continuous line; computed curve: dotted line. The computed flow curve is obtained from a 'family' of couples of VBN parameters (detrusor force $\mathrm{k}$ and prostatic urethral counter-pressure pucp) which implies different detrusor pressure (pdet) curves (equivalent voids); two examples: $k=1.0$ - pucp = $16 \mathrm{~cm} \mathrm{H}_{2} \mathrm{O}$, and $k=2.3-$ pucp $=62 \mathrm{~cm} \mathrm{H}_{2} \mathrm{O}$. b Four set of possible obstructive status. Each curve represents a family of equivalent voids. The $\mathrm{D}$ value (in $\mathrm{cm}_{2} \mathrm{O}$ ) is the pucp value at the intercept of a curve with the reference line $k=1.0$.

number which represents the urethral compression (in $\mathrm{cm} \mathrm{H}_{2} \mathrm{O}$ ) due to $\mathrm{BPH}$, assuming a normal detrusor $(k=1)$ and resulting in a computed flow curve which superimposes the recorded flow curve. Another strength of the D index is that it is independent of the volume voided when that volume is over $100 \mathrm{ml}$ and also independent of perturbations of short duration that can occur during the flow. The value of the $\mathrm{D}$ index ranges from 0 to 60 with the highest values implying near acute urinary retention.

\section{Population and Urodynamic Studies}

Urodynamic tracings (FF and PF) of 30 non-neurological BPH patients before TURP and of 30 men suspected of BPH included in a medical therapy trial were retrospectively analyzed. Mean age was $66 \pm 11$ years (range 50-85 years) in the surgical group and $66 \pm 7$ years (range 54-76 years) in the medical therapy group. PF was performed standing, with a $6 \mathrm{~F}$ double lumen urethral catheter in situ, after cystometry at filling rate of $50 \mathrm{ml} / \mathrm{min}$ (normal saline at room temperature). Rectal pressure was measured using a punctured balloon filled with $2 \mathrm{ml}$ of saline solution at room 
Table 1. Comparison of A-G number classification and D index findings

\begin{tabular}{llll}
\hline D index & A-G number & Class & Number of patients \\
\hline$>32.5$ & $>40$ & true positive & 32 \\
$<32.5$ & $>40$ & false negative & 7 \\
$>32.5$ & $<40$ & false positive & 7 \\
$<32.5$ & $<40$ & true positive & 14 \\
\hline
\end{tabular}

Table 2. Evaluation of D index for the 2 sub-populations

\begin{tabular}{llllrr}
\hline Population & Sensitivity & PPV & Specificity & NPV & LR+ LR- \\
\hline Pre-TURP $(\mathrm{N}=30)$ & $83.3 \%$ & $75.0 \%$ & $58.3 \%$ & $70.0 \%$ & 1.99 \\
Before inclusion in a medical therapy trial $(\mathrm{N}=30)$ & $80.9 \%$ & $89.4 \%$ & $77.7 \%$ & 6.28 \\
\hline
\end{tabular}

$\mathrm{PPV}=$ Positive predictive value NPV= negative predictive value

temperature in order to avoid pressure artifacts. Detrusor pressure was the difference vesical minus rectal pressure. FF was recorded privately.

Urodynamic studies recordings were performed using Aquarius from Laborie in the pre-TURP population and Menuet from Medtronic in the medical therapy group.

Tracings were reviewed independently by 2 investigators in order to evaluate A-G number. In case of discrepancy between the 2 values ( $6 \%$ of the tracings), a session with joint interpretation led to a final decision. Criteria for exclusion were a voided volume $<100 \mathrm{ml}$ and/or tracings with an interrupted flow.

\section{Criteria for $\mathrm{BOO}$}

Using the Abrams-Griffiths number, a patient was categorized as obstructed $\mathrm{PF} A-\mathrm{G}>40 \mathrm{~cm} \mathrm{H}_{2} \mathrm{O}$, equivocal $\mathrm{PF} 40 \mathrm{~cm} \mathrm{H}_{2} \mathrm{O}$ $\geq A-G \geq 20 \mathrm{~cm} \mathrm{H}_{2} \mathrm{O}$, and unobstructed PF A-G $<20 \mathrm{~cm} \mathrm{H}_{2} \mathrm{O}$. A-G number was derived from PF recordings. A-G and mPIP are respectively strongly correlated to pucp and $k$ [8]:

$\mathrm{A}-\mathrm{G} \sim 1.41 *$ pucp -6.18

$\mathrm{mPIP} \sim 24.5^{*} k+52.9$

The ICS criteria for obstruction are easily translated for pucp. Thus, using the $\mathrm{D}$ index, a patient would be obstructed if $\mathrm{D}>32.5$ $\mathrm{cm} \mathrm{H}_{2} \mathrm{O}$, equivocal if $32.5 \mathrm{~cm} \mathrm{H}_{2} \mathrm{O} \geq \mathrm{D} \geq 18.5 \mathrm{~cm} \mathrm{H}_{2} \mathrm{O}$ and unobstructed if $\mathrm{D}<18.5 \mathrm{~cm} \mathrm{H_{2 }} \mathrm{O}$. D index was obtained from all tracings of a given patient (mean value in a session).

\section{Evaluation of $\mathrm{BOO}$}

For each patient, comparison was made between A-G and D classification.

\section{Statistical Analysis}

Demographic data are given as mean \pm standard deviation (SD). Sensitivity was defined as the ratio between true positives
(BPH patient with positive test) and the sum true positives plus false negatives (BPH patient with negative test), and identified those patients with BPH. Specificity was defined as the ratio between true negatives (patient without BPH and negative test) and the sum true negatives plus false positives (patient without BPH and positive test), and identified those patients without BPH. The positive predictive value was defined as the ratio between true positives and the sum true plus false positives, to answer the likelihood of a patient having BPH given that the test result was positive.

The negative predictive value was defined as the ratio between true negatives and the sum true plus false negatives and addressed the likelihood that a patient did not have BPH given when the test result was negative. The likelihood ratio positive $(\mathrm{LR}+)$ was the ratio between Sensitivity and ( 1 - Specificity), and was defined as how much more likely a patient who tests positive to have BPH compared to one who tests negative. The likelihood ratio negative (LR-) was the ratio between ( 1 - Sensitivity) and Specificity, and was defined as how much more likely a patient who tests negative to have BPH compared to one who tests negative.

\section{Results}

Comparison of A-G number classification and D index findings are presented in table 1.

For the whole population, the results are described hereafter.

Sensitivity, which is the probability for a patient to be obstructed when $\mathrm{D}>32.5 \mathrm{~cm} \mathrm{H}_{2} \mathrm{O}$ and the positive 
predictive value, which is the probability to observe $\mathrm{D}>$ $32.5 \mathrm{~cm} \mathrm{H} \mathrm{H}_{2} \mathrm{O}$ when a patient is obstructed, had the same value $82.05 \%$. Specificity, which is the probability for a patient to be equivocal or unobstructed when $\mathrm{D} \leq 32.5$ $\mathrm{cm} \mathrm{H}_{2} \mathrm{O}$, and the negative predictive value, which is the probability to observe $\mathrm{D}>32.5 \mathrm{~cm} \mathrm{H}_{2} \mathrm{O}$ when a patient is equivocal or unobstructed, had the same value $66.66 \%$. An analysis of the two sub-populations is given in table 2.

\section{Discussion}

BOO is a common condition in aging man suffering from the consequences of BPH. Urodynamic studies have two basic aims: to reproduce the patient's lower urinary tract symptoms and to better understand the underlying pathophysiology of these complaints. Currently, the A-G number deduced from PF represents the "gold standard" to diagnose and quantify BOO. Single urodynamic methods of diagnosing BOO noninvasively have been proposed to be an alternative to invasive PF: uroflowmetry with only maximum flow rate [12], penile compression and release (PCR) index [13], penile cuff test (modified nomogram) [14], external condom [15], and Doppler urodynamics [16]. Among these, PCR index is easy to perform and shows $91 \%$ sensitivity with $70 \%$ sensitivity [13]. All other methods have limitation. Sensitivity of maximum flow rate less than $10 \mathrm{ml} / \mathrm{s}$ remains poor (47\%) [12]. External condom has a large failure rate, reported as $25 \%$ by Pel et al. [15] and shows $64 \%$ sensitivity with $79 \%$ specificity. The penile cuff test shows $73 \%$ sensitivity with $75 \%$ specificity [14] and needs combination of 3 parameters (flow rate, cuff pressure and PCR index) to provide $86 \%$ sensitivity with $87 \%$ specificity [14]. Doppler ultrasound seems to give valuable results: $78 \%$ sensitivity with $92 \%$ specificity [16]; in fact the technique suffers from several problems: difficulty to locate the sites where flow velocity is measured, expensive equipment to perform, patient's cooperation and sitting position for voiding (most men void while standing).

The $\mathrm{D}$ index is proposed as an alternative to invasive urodynamic studies because it can be obtained from a FF. In addition, it is independent of initial bladder volume and of circumstantial factors such as non repeatable changes of nervous control during voiding (delayed sphincter opening, fading of detrusor excitation) which might affect the value of Qmax [2].

In current pratice, evaluation of $\mathrm{D}$ index can be obtained from a simple device which associates a flowme- ter and a "click and read" version of the $\mathrm{VBN}^{\circledR}$ software. Such a simple device can be used without a complex and expensive equipment and can help for quick screening and watchful waiting of BPH patients.

The use of D index has a high sensitivity ( 83.3 vs. $80.9 \%)$ and a high PPV (75.0 vs. $89.4 \%)$ in the subgroups analyzed in this comparative study, suggesting that obstructed patients can be correctly identified. False negatives and false positives of the $\mathrm{D}$ index could be the consequence of its inability to distinguish between two scenarios: a severe obstruction with high detrusor contractility vs. a mild obstruction with weak detrusor contractility which both results in a low Qmax. When the 7 false positives were analyzed with the VBN model, 4 were identified as resulting from a low detrusor contractility condition.

Specificity and NPV have a relatively lower value. That result could be the consequence of a biased recruitment: one half of the patients are BPH patients who were tested before TURP while the other half were only suspected of having BPH.

\section{Conclusion}

In this limited clinical series, the D index which can be obtained from a free uroflow appears as a valuable alternative to invasive urodynamic investigations. Evaluation of $\mathrm{D}$ index is noninvasive, cheap and do not requires sophisticated equipment, thus is able to allow a quick screening out of a dedicated urodynamic department. Then, the D index can be useful when the diagnosis of BOO needs to be more solidly established before a treatment decision or in men suspected of BPH who elect for watchful waiting. However, though the association with the A-G number that is used to define BOO appears acceptable, the D index cannot substitute for the knowledge of the voiding pressure if that parameter is important in the ultimate treatment decision.

\section{Acknowledgements}

We thank Derek J. Griffiths PhD (Division of Geriatric Medicine, University of Pittsburg, PA, USA) and Jean-François Hermieu MD (Department of Urology, Hôpital Bichat, Paris, France) who has kindly supplied us with pressure-flow records. We thank also Smith Kline Beecham who allowed us to use the curves obtained in some clinical trials. 


\section{References}

1 Griffiths DJ, Höfner K, van Mastrigt R, Rollema HJ, Spånberg A, Gleason D: Standardization of terminology of lower urinary tract function: pressure-flow studies of voiding, urethral resistance and urethral obstruction. Neurourol Urodyn 1997;16:1-18.

$>2$ Valentini FA, Nelson PP, Besson GR, Zimmern PE: Challenging the maximum flow rate: a new index of voiding dysfunction in men with benign prostatic enlargement. BJU Int 2008;101:995-999.

-3 Valentini FA, Gilchrist A, Nelson PP, Zimmern PE: A new index of voiding dysfunction in men with benign prostatic hyperplasia undergoing transurethral resection of the prostate adds superior information to the traditional maximum flow rate (Qmax). Curr Urol 2011;5:151-157.

-4 Valentini FA, Besson GR, Nelson PP, Zimmern PE: A mathematical micturition model to restore simple flow recordings in healthy and symptomatic individuals and enhance uroflow interpretation. Neurourol Urodyn. 2000;19:153-176.

5 Griffiths DJ: Urodynamics: the mechanics and hydrodynamics of the lower urinary tract. Bristol: Adam Hilger, 1980.
6 Griffiths DJ: The mechanics of the urethra and of micturition.Br J Urol 1973;45:497507.

7 Besson G, Valentini F, Nelson P: Progress in the theory of flow through the urethra during micturition; in Barbalias GA (ed): International Continence Society 26th Annual Meeting. Bologna, Monduzzi, 1996, pp39-43.

8 Valentini FA, Mazières L, Nelson PP: Can modeled analysis of urodynamic recordings help to demonstrate the nervous control of bladder and urethra during micturition? UroToday Int J 2010;3.

9 Valentini FA, Griffiths DJ, Zimmern PE, Besson GR, Nelson PP: Quantification de l'obstruction urétrale chez l'homme: comparaison des méthodes usuelles avec la méthode VBN ${ }^{\circledast}$. Ann Réadap Med Phys 2005; 48:11-19.

10 Schäfer W, Sterling AM: 1995 Simple analysis of voiding function by coefficient (OCO) and detrusor contraction coefficient (DECO). Abstract book, ICS 25th Annual Meeting (Sydney), 388-389.

11 Griffiths DJ, Constantinou CE, van Mastrigt $\mathrm{R}$ : Urinary bladder function and its control in healthy females. Am J Physiol 1986;251: R225-230.
12 Reynard JM, Yang Q, Donovan JL, Peters TJ, Schafer W, de la Rosette JJ, Dabhoiwala NF, Osawa D, Lim AT, Abrams P: The ICS'BPH' study: uroflowmetry, lower urinary tract symptoms and bladder outlet obstruction. Br J Urol 1998;82:619-623.

13 Sullivan MP, Yalla SV: Penile urethral compression-release maneuver as a non-invasive screening test for diagnosing prostatic obstruction. Neurourol Urodyn 2000;19:657669

14 Drinnan M, Harding C, Blake C, McIntosh S, Robson WW, Pickard R, Abrams P, Ramsden P, Griffiths C: Combination of non-invasive urodynamic parameters from a single penile cuff test for diagnosis of bladder outlet obstruction (abstract). Joint meeting ICSIUGA, 34th Annual Meeting, Paris, France, August 25-27, 2004.

15 Pel JJ, Bosch JL, Blom JH, Lycklama a Nijeholt AA, van Mastrigt R: Development of a non -invasive strategy to classify bladder outlet obstruction in male patients with LUTS. Neurourol Urodyn 2002;21:117-125.

16 Ozawa H, Chancellor MB, Ding YY, Nasu Y, Yokoyama T, Kumon H: Noninvasive urodynamic evaluation of bladder outlet obstruction using Doppler ultrasonography. Urology 2000;56:408-412. 